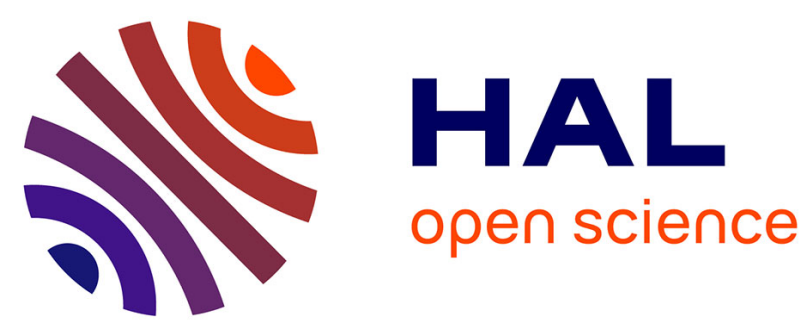

\title{
Determination of the Vinyl Fluoride line intensities by TDL spectroscopy: the Object Oriented approach of Visual Line Shape Fitting Program to the line profile analysis.
}

Nicola Tasinato, Andrea Pietropolli Charmet, Paolo Stoppa, Santi Giorgianni

\section{To cite this version:}

Nicola Tasinato, Andrea Pietropolli Charmet, Paolo Stoppa, Santi Giorgianni. Determination of the Vinyl Fluoride line intensities by TDL spectroscopy: the Object Oriented approach of Visual Line Shape Fitting Program to the line profile analysis.. Molecular Physics, 2010, 108 (06), pp.677-685. 10.1080/00268970903468305. hal-00588657

\section{HAL Id: hal-00588657 https://hal.science/hal-00588657}

Submitted on 26 Apr 2011

HAL is a multi-disciplinary open access archive for the deposit and dissemination of scientific research documents, whether they are published or not. The documents may come from teaching and research institutions in France or abroad, or from public or private research centers.
L'archive ouverte pluridisciplinaire $\mathbf{H A L}$, est destinée au dépôt et à la diffusion de documents scientifiques de niveau recherche, publiés ou non, émanant des établissements d'enseignement et de recherche français ou étrangers, des laboratoires publics ou privés. 


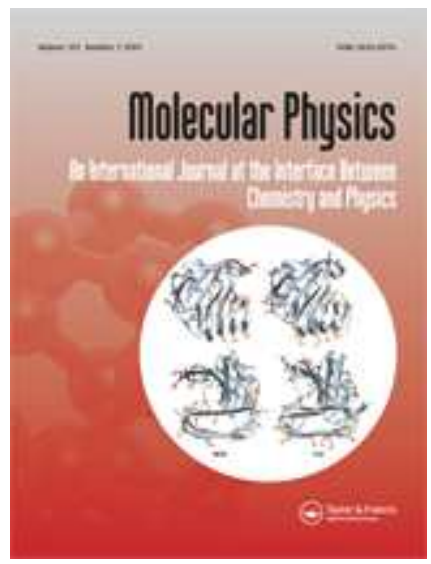

Determination of the Vinyl Fluoride line intensities by TDL spectroscopy:

the Object Oriented approach of Visual Line Shape Fitting Program to the line profile analysis.

\begin{tabular}{|r|l|}
\hline Journal: & Molecular Physics \\
\hline Manuscript ID: & TMPH-2009-0298.R1 \\
\hline Manuscript Type: & $\begin{array}{l}\text { Special Issue Paper -HRMS Stabia 09/ High Resolution Molecular } \\
\text { Spectroscopy }\end{array}$ \\
\hline Date Submitted by the \\
Author: & 20-Oct-2009 \\
\hline Keymote List of Authors: & $\begin{array}{l}\text { TASINATO, Nicola; Universita' Ca' Foscari di Venezia, Dipartimento } \\
\text { Chimica Fisica } \\
\text { PIETROPOLLI CHARMET, Andrea; Universita' Ca' Foscari di Venezia, } \\
\text { Dipartimento di Chimica Fisica } \\
\text { STOPPA, Paolo; Universita' Ca' Foscari Venezia, Chimica Fisica } \\
\text { GIORGIANNI, Santi; University of Venice }\end{array}$ \\
\hline & $\begin{array}{l}\text { Vinyl fluoride, Line parameter determination, Line shape fitting } \\
\text { software, Weak and strong collision models, Voigt and Dicke } \\
\text { narrowed profiles }\end{array}$ \\
\hline & \\
\hline
\end{tabular}

\section{s ScholarONE" \\ Manuscript Central}




\title{
Determination of the Vinyl Fluoride line intensities by TDL spectroscopy: the Object Oriented approach of Visual Line Shape Fitting Program to the line profile analysis
}

\author{
Nicola Tasinato ${ }^{*}$, Andrea Pietropolli Charmet, Paolo Stoppa, Santi Giorgianni
}

Università Ca' Foscari di Venezia - DCF - I-30123 Venezia (Italy)

\begin{abstract}
In this work the self-broadening coefficients and the integrated line intensities for a number of ro-vibrational transitions of vinyl fluoride have been determined for the first time by means of TDL spectroscopy. The spectra recorded in the atmospheric window around $8.7 \mu \mathrm{m}$ appear very crowded with a density of about 90 lines per $\mathrm{cm}^{-1}$. In order to fit these spectral features a new fitting software has been implemented. The program which is designed for laser spectroscopy, can fit many lines simultaneously on the basis of different theoretical profiles (Doppler, Lorentz, Voigt, Galatry and Nelkin Ghatak). Details of the Object Oriented implementation of the application are given. The reliability of the program is demonstrated by determining the line parameters of some ro-vibrational lines of sulphur dioxide in the $v_{1}$ band region around $9 \mu \mathrm{m}$. Then the software is used for the line profile analysis of vinyl fluoride. The experimental line shapes show deviations from the Voigt profile which can be well modelled by using a Dicke narrowed line shape function. This leads to the determination of the selfnarrowing coefficient within the framework of the strong collision model.
\end{abstract}

Keywords: Vinyl fluoride; Line parameter determination; Line shape fitting software; Weak and strong collision models; Voigt and Dicke narrowed profiles.

\footnotetext{
* Corresponding author.

E-mail: tasinato@unive.it; Phone: +39 041 2348598; Fax: +39 0412348594

Address: Università Ca' Foscari Venezia, Dipartimento di Chimica Fisica, Dorsoduro 2137, I-30123 VENEZIA (Italy)
} 


\section{Introduction}

The study of the Earth and planetary atmospheres and of the interstellar medium by means of spectroscopic techniques has rapidly grown up since the earlier experiments in the second half of the 1900. Among the various techniques, infrared spectroscopy plays a significant role, in particular for remote sensing of the terrestrial atmosphere. In fact, IR remote sensing permits to monitor and accurately retrieve the concentration of the atmospheric constituents and trace pollutants. Though, in order to obtain reliable measurements, an accurate knowledge of the line parameters, such as line centres, intensities and pressure broadening coefficients, is required.

Thanks to the availability of always more powerful computers, the time consuming task of inverting the experimental spectra to extract spectroscopic parameters has become increasingly assisted by computer programs. Compared to the huge number of software available for extracting the Hamiltonian's parameters from the assigned line positions (a list of freely available devices is given in [1]), some programs specifically designed to perform the line shape analysis by fitting the experimental spectrum have been presented (see, for example, $[2-6]$ ). It is worthwhile to note that these programs appear specifically designed to deal mostly with FTIR spectra. As a consequence, during the fitting procedure, they explicitly take into account the step of the parameterization of the FTIR instrumental line shape. These software, based on the multi-spectrum fitting technique, are the best choice when the large number of lines recorded by the interferometer in a single scan needs to be analyzed.

On the other hand, the spectrometers based on laser sources have a simpler instrumental contribution, generally modelled as a Gaussian or Lorentzian function. 
Furthermore, the spectral region which can be recorded in a scan is of the order of some wavenumbers, thus greatly reducing the amount of lines to be accounted for in a single spectrum. In many cases, the line shape analysis is carried out by using home made programs which are sometimes designed to fit the experimental features to a single theoretical function. Further, some programs are able to fit only one absorption line at time and still run from a command line window, thus avoiding an immediate comparison between the experimental data and the theoretical model. Commercial packages, such as IGOR Pro [7] and Origin [8], can be employed as well, although they are not completely well suited for this purpose.

Vinyl Fluoride, $\mathrm{CH}_{2} \mathrm{CHF}$, is widely used by industry mainly as monomer for the production of synthetic materials such as poly-vinyl fluoride. The potential role of halogenated alkenes as air pollutants has motivated a number of spectroscopic investigations and studies about their reactivity toward hydroxyl radical and ozone (see Refs. $[9,10]$ and references therein).

The infrared spectra of vinyl fluoride have been deeply investigated by our research group (see Refs. [11 - 13] and references therein). The studies have mainly dealt with the analysis of the high resolution spectra and the obtained results have led to the determination of accurate spectroscopic parameters for a number of fundamental and overtone vibrations and a thorough understanding of the observed perturbations. Recently, the medium resolution spectrum has been studied in the $400-8000 \mathrm{~cm}^{-1}$ region and the integrated absorption cross sections have been determined for the first time [13]. In addition, ab-initio calculations with large basis sets have been performed at the $\operatorname{CCSD}(\mathrm{T})$ level of theory leading to the determination of harmonic and anharmonic force fields. 
Despite the industrial importance of vinyl fluoride, there exist no studies on the determination of the line parameters of the ro-vibrational absorption lines. The lack of literature data is partly motivated by the complex structure of the high resolution spectrum. In the present work we report the very first results obtained from the line shape analysis in the $v_{7}$ band region around $1148 \mathrm{~cm}^{-1}$ by means of tunable diode laser (TDL) spectroscopy. In this region, the spectrum of vinyl fluoride appears very crowded even at low pressures. Hence, the analysis strongly requires a fitting software capable to simultaneously fit all the absorptions present in the spectral micro-windows acquired by the TDL system. For this reason we implemented a new software for the line shape fitting and the retrieval of the line parameters. The program, called Visual Line Shape Fitting Program (VLSFP), can fit several lines at once using different theoretical line shape functions. These include the Doppler, Lorentz and Voigt profiles, as well as the Galatry and Nelkin - Ghatak functions. The reliability of VLSFP has been tested by retrieving the sulphur dioxide line parameters and comparing them with the literature values. In this paper we also describe the program implementation and validation. In Section 2 the adopted experimental procedure is outlined while Sections 3 and 4 are concerned with the description of the fitting software and its test. Then in Section 5 we illustrate the results of the line shape analysis of vinyl fluoride. Conclusions and future work are addressed in Section 6.

\section{Experimental procedure and data inversion}

The high resolution spectra of either sulphur dioxide or vinyl fluoride were acquired on our tunable diode laser spectrometer employing a $92.3( \pm 0.2) \mathrm{cm}$ path 
length cell with $\mathrm{KBr}$ windows. The gas sample pressures were measured employing an Alcatel ARD 1003 capacitance gauge with a full scale range of $1000 \mathrm{~Pa}$ and a quoted manufacturer's accuracy of 1.5 Pa. Before each measurements the cell was evacuated down to about $10^{-4} \mathrm{~Pa}$; an elapse time of 10 and 20 minutes, for $\mathrm{SO}_{2}$ and $\mathrm{CH}_{2} \mathrm{CHF}$ respectively, between the filling of the cell and the spectrum acquisition was adopted. The spectra were calibrated employing the $\mathrm{SO}_{2}$ line frequencies obtained by high resolution FTIR measurements. The $\mathrm{SO}_{2}$ and $\mathrm{CH}_{2} \mathrm{CHF}$ gas samples were provided by Sigma - Aldrich (99.9\% purity) and Peninsular Chemical Research Inc. (99\% purity), respectively, and were used without any further purification; no buffer gasses were employed. The $\mathrm{SO}_{2}$ spectra were acquired at $297( \pm 1) \mathrm{K}$ and the pressures were varied in the range between 15 and $800 \mathrm{~Pa}$. The measurements on $\mathrm{CH}_{2} \mathrm{CHF}$ were carried out at $299( \pm 1) \mathrm{K}$ with pressures ranging from 7 to $68 \mathrm{~Pa}$.

Since the instrumental response of our TDL spectrometer is well described by a Gaussian function, its contribution to the line shapes was explicitly taken into account during the fitting procedure by fixing the Doppler half width to an effective value, $\gamma_{D}^{e f f}$, given by

$$
\gamma_{D}^{e f f}=\sqrt{\left(\gamma_{D}^{\text {sample }}\right)^{2}+\left(\gamma_{D}^{T D L}\right)^{2}}
$$

where $\gamma_{D}^{\text {sample }}$ and $\gamma_{D}^{T D L}$ are the molecular and instrumental Doppler half widths, respectively. The value of $\gamma_{D}^{\text {eff }}$ was retrieved by fitting the spectral lines recorded at low pressure (less than $30 \mathrm{~Pa}$ and $10 \mathrm{~Pa}$ for $\mathrm{SO}_{2}$ and $\mathrm{CH}_{2} \mathrm{CHF}$, respectively) to a Gaussian profile. 


\section{Visual Line Shape Fitting Program implementation: an Object Oriented approach}

VLSFP has been written combining Microsoft Visual Basic and C\# for the graphical interface and the computational kernel, respectively. The determination of the line parameters within a given theoretical model is performed through an iterative procedure using the Levenberg - Marquardt algorithm $[14,15]$. The algorithm has been implemented following the pseudo-code described in [16] and then modified in order to set bounds to the variability of the parameters or to keep the parameter values constrained, as described by Kanzow et al. [17]. In the current implementation, the algorithm terminates when the maximum number of iterations is completed or when one of the convergence criteria is met. These criteria are related to the square of the Euclidean norm of the residuals, its gradient and to the variation of the parameters between two subsequent iterations. The fitting routine runs as a background process avoiding the program interface to be frozen during the execution of the fitting task.

The computational kernel, schematically depicted in Figure 1, has been designed following an Object Oriented Programming approach [18]. This programming technique, born around 1960 s, uses objects and their interactions to design computer programs. An application is then composed of a collection of cooperating objects, rather than an ensemble of subroutines that compute specific tasks. Each box of Figure 1 is referred to as class and it represents an "object". The class defines the properties (the class variables) of the corresponding object and its behaviour (the class methods). The object is able to process data and mostly to interact with other objects by receiving and sending messages. In a pictorial representation, every single object can be seen as a little 
machine with its own task. The set of distinct machines then cooperate to accomplish an objective: in our case the fit of the experimental line shape by means of different theoretical profiles.

Referring to Figure 1, the classes Vector, Matrix and Complex implement vector, matrix and complex algebra, respectively. They are used by the other objects of the project to store and process the data. The matrices have been implemented as onedimensional arrays thus speeding up the access to their elements. The fitting routines have been encapsulated inside the class LevMar and they have been designed to deal with a generic object of the type Line Shape. Line Shape represents an abstract class, that is an abstract object which defines the common characteristics possessed by all the line shapes (for example, the computed line profile, the line parameters array and the Jacobian matrix). The single line shape functions have been derived from the main abstract class and within each class a specific implementation of the methods has been made. As a consequence, new line shape functions, such as the speed dependent profiles, can be implemented without changing neither the LineShape nor the LevMar classes.

The line shape functions have been implemented in the normalized form. Denoting the generic line function by $f(\mathbf{p})$, it is normalized to unitary area:

$$
\int_{-\infty}^{+\infty} f(\mathbf{p}) d x=1
$$

where $\mathbf{p}=[x, y, z, \ldots]$ is the vector of dimensionless parameters which are described in Table 1 following the notation of Varghese and Hanson [19]. As it can be seen, the line parameters are normalized by the effective Doppler half width defined as 


$$
\alpha_{D}=\frac{\gamma_{D}}{\sqrt{\ln 2}}
$$

where $\gamma_{D}$ is the dimensional Doppler half width given by [20]

$$
\gamma_{D}=\frac{v_{0}}{c}\left(\frac{2 k_{\mathrm{B}} N_{\mathrm{A}} T \ln 2}{m}\right)^{1 / 2}
$$

where $v_{0}$ is the resonant frequency; $c$ is the speed of light; $k_{\mathrm{B}}$ is the Boltzman's constant; $N_{\mathrm{A}}$ is the Avogadro's number; $T(\mathrm{~K})$ is the absolute temperature, $m$ (a. m. u.) is the mass of the absorbing gas.

According to Beer - Lambert's law, the transmission of monochromatic radiation travelling a path of length $\ell$ through a uniform gas at pressure $p$ is given by

$$
I(v)=I_{0} \mathrm{e}^{-k(v) p \ell}
$$

where $I_{0}$ is the unattenuated intensity of the radiation having frequency $v$. The quantity $k(v)$ is called the absorption coefficient and, for a single absorption line, it can be expressed in the form:

$$
k(v)=S f\left(v-v_{0}\right)
$$


where $S$ is the integrated absorption coefficient (also referred to as line intensity) and $f\left(v-v_{0}\right)$ is the line shape function as defined in equation (2). The dimensionless line shape functions implemented in VLSFP are listed in Table 2. During the fitting procedure, VLSFP refines the line intensity $S$, the line frequency centre $v_{0}$ and the remaining line parameters, which clearly depend on the specific line shape function.

The Voigt profile is widely used for modelling the spectral line profiles and in other scientific areas ([21] and references therein), and hence the issue of its computation has been dealt over long time and intensive efforts have led to the development of many algorithms [22 - 27]. A comprehensive survey of the different computational approaches has been reported by Schreier [28]. In VLSFP, the Voigt function is computed employing the optimized version of Humliček's code proposed by Wells [29]. Since this algorithm computes also the imaginary part of the complex probability function, it can be conveniently used for the computation of the Nelkin Ghatak profile [30, 31], as well.

Concerning the Galatry function [32], the profile is computed by the Fourier transform of the corresponding correlation function, $\Phi(y, z, t)$, following the algorithm proposed by Ouyang and Varghese [33]:

$$
f(x-s, y, z)=\frac{1}{\pi} \operatorname{Re}\{\Im[\Phi(y, z, t)]\}
$$

where $\mathfrak{I}$ indicates the Fourier transform operator. The derivatives are in turn computed via the Fourier transform of the appropriate correlation function. As a consequence, a discrete fast Fourier transform routine (DFFT) has been implemented. 


\section{Test of VLSFP with $\mathrm{SO}_{2}$ lines}

VLSFP has been tested by retrieving the parameters of some ro-vibrational lines of sulphur dioxide in the region around $9 \mu \mathrm{m}$. This region is characterized by the absorptions of the $v_{1}$ fundamental corresponding to the symmetric stretching normal mode. In this section the results for a number of lines are presented in order to demonstrate the reliability of the application.

An example of the fits obtained for an isolated absorption line is given in Figure 2. When fitted to a Voigt profile, this line seems to show the effect of Dicke narrowing as suggested by the shape of the residuals. As a consequence, the line has also been fitted employing the weak and strong collision models. As can been seen from the plots of the residuals, the models accounting for the velocity changing collision effects reproduce properly the observed line shape.

A wider spectral region is shown in Figure 3. In this case, the lines have been fitted to a Voigt profile, employing the multi - line fitting procedure. In general, all the lines have been well reproduced although the residuals show some regular trends. This behaviour can be partly explained by the presence of very weak lines underneath the stronger ones. These lines, being not resolved at all, can not be taken into account during the fits but at the same time cause asymmetries in the observed absorptions. For the same reason, the Dicke narrowed profiles are not able to account for all the deviations. Anyway, even in such situation, the physical meaning of the parameters is preserved. This has been confirmed by plotting the collisional half widths and the line intensities as a function of the gas pressure: the line parameters lie on straight lines passing through the origin. 
The obtained line parameters are listed in Table 3 together with the more recent literature values $[34,35]$ whose comparison is shown in Figure 4 . As it can be seen, the present data and the literature values agree in a very satisfactory manner, indicating the reliability of the retrieved line parameters and hence validating the computational kernel of VLSFP.

\section{Determination vinyl fluoride line shape parameters}

The spectrum of vinyl fluoride in the $8.7 \mu \mathrm{m}$ atmospheric region is characterized by the ro-vibrational transitions belonging to the $v_{7}$ band which corresponds to the $\mathrm{C}-\mathrm{F}$ stretching. As pointed out in the introduction, this spectral region is very crowded: the high density of lines, about 90 lines per $\mathrm{cm}^{-1}$, can be appreciated in Figure 5 (a). Besides the stronger absorption of the $v_{7}$ normal mode, the majority of the weakest lines is due to the ro-vibrational transitions of the $v_{7}+v_{9}-v_{9}$ hot band. Due to their intensity these lines strongly affect the main features and therefore they must be accounted during the fitting procedure.

When the spectral lines are fitted to the Voigt profile, the shape of the residuals shows significant deviations from this model, as illustrated in Figure 5 (b). For this reason the lines have also been fitted employing the Nelkin - Ghatak profile. By using this strong collision model there is a dramatic improvement of the residuals as it is shown in Figure 5 (c): the standard deviation of the fit decreases by about a factor of two. Therefore, in the pressure range investigated the self-broadened vinyl fluoride sample appears as system strongly affected by the motional narrowing effect in which 
either the velocity changing collisions and the internal state - dephasing collisions play an almost equal role in determining the shape of the spectral lines. Consequently, the line profiles accounting for the Dicke narrowing effect must be employed to properly model the experimental features.

The retrieved line parameters for the $v_{7}$ lines are listed in Table 4 . The line parameters of the hot band have been determined as well, but they are not given here because of the lack of a reliable assignment of the ro-vibrational transitions at present. Again, we preferred to not report the self-broadening coefficient of three lines because their accuracy is too low. This is due to the small range of vinyl fluoride pressures adopted in the experiments which limits the amount of pressure broadening for some of the lines. On the other hand, the use of low pressures is strongly recommended in order to avoid the saturation of the absorptions, and hence working in the experimental conditions of applicability of the Beer - Lambert law. As can be seen from Table 4, the obtained self-broadening coefficients ranges from 0.4 to $0.9 \mathrm{~cm}^{-1} \mathrm{~atm}^{-1}$. Such a wide interval is shown, for instance, by water vapour, whose self-broadening parameters are between 0.1 and $0.5 \mathrm{~cm}^{-1} \mathrm{~atm}^{-1}$ [36]. Besides, to a first investigation no dependence on the quantum numbers have been observed. This is understandable by considering the reduced set of data in which the majority of lines belong to the ${ }^{\mathrm{Q}} \mathrm{P}$ subband. Therefore, in order to draw a more reliable conclusion about the quantum number dependence of the self-broadening coefficients, the analysis need to extended to a spectral region as wide as possible including the B-type transitions, as well.

The fits employing the Nelkin - Ghatak profile led to the determination of the narrowing parameter which result to be $0.40( \pm 0.08) \mathrm{cm}^{-1} \mathrm{~atm}^{-1}$. As can be seen, the magnitude of the self-broadening and self-narrowing have a similar extent. 


\section{Conclusions and Outlook}

The very first results of the line shape analysis of the vinyl fluoride infrared absorptions have been presented and discussed. In addition, the details of the implementation and validation of the fitting software (VLSFP) used for the analysis have been given.

The program has been specifically designed to deal with the spectra recorded by means of laser based spectrometers in which the instrumental function can be modelled as a Gaussian or Lorentzian functions. Besides these two functions, the spectral absorptions can be fitted employing the Voigt profile as well as the weak and strong collision models which account for the Dicke narrowing effect. The application has been implemented following a strongly Object Oriented Programming approach which leads to an easier maintenance and future developments. The program runs under Windows environment and it has a standard graphical interface according to the version of the operation system currently in use. In order to test the program, the line parameters of some sulphur dioxide lines have been determined and compared with the literature data. Once validated, VLSFP has been used to obtain the line parameters of $\mathrm{CH}_{2} \mathrm{CHF}$.

The vinyl fluoride spectra in the $1148 \mathrm{~cm}^{-1}$ atmospheric region have been recorded with our TDL spectrometer at $299 \mathrm{~K}$. The measurements have been carried out on a self-broadened $\mathrm{CH}_{2} \mathrm{CHF}$ sample with pressures in the range between 7 and $68 \mathrm{~Pa}$ : even at these low gas pressures the spectrum presents a high density of absorption lines. The self-broadening and integrated absorption coefficients for 13 lines belonging to the $\mathrm{R}$ branch of the $v_{7}$ normal mode have been determined for the first time. The experimental line shapes showing deviations from the Voigt profile have also been 
fitted using the Nelkin - Ghatak profile. This has led to the determination of the self narrowing coefficient.

The present paper gives an overview of the work that we are carrying out at present. Indeed, on the basis of the good results obtained from the analysis of the vinyl fluoride crowded spectra, we are going to extend the investigation in a spectral region as wide as possible with the aim to derive the vinyl fluoride line parameters in the $8-9$ $\mu \mathrm{m}$ atmospheric window. Future work will also be devoted toward a definitive assignment of the transitions belonging to the $v_{7}+v_{9}-v_{9}$ hot band and to an improvement of the accuracy for some of the broadening coefficients.

\section{Acknowledgments}

The authors thank Prof. P. L. Varghese for the code for the computation of the Galatry function. The authors also gratefully acknowledge Prof. J. M. Flaud for the $\mathrm{SO}_{2}$ data about $v_{1}$ and $v_{1}+v_{2}-v_{2}$ upon which the assignments have been made. This work was financially supported by PRIN 2007 (project: "Trasferimenti di energia, carica e molecole in sistemi complessi"). 


\section{References}

[1] Z. Kisiel, PROSPE - Programs for Rotational SPEctroscopy, <http:// info.ifpan.edu.pl/ kisiel/prospe.htm/>.

[2] M. Carlotti, Appl. Opt. 27, 3250 (1988).

[3] D.C. Benner, C.P. Rinsland, V.M. Devi, M.A.H. Smith, D.A. Atkins, JQSRT 53, 705 (1995).

[4] A.S. Pine, T. Gabard, JQSRT 66, 69 (2000).

[5] D. Jacquemart, J.Y. Mandin, V. Dana, N. Picqué, G.A. Guelachvili, Eur. Phys. J. D 14, 55 (2001).

[6] J.J. Plateaux, L. Ragalia, C. Boussin, A. Barbe, JQSRT 68, 507 (2001).

[7] Wavemetrics Inc. <http://www.wavemetrics.com>.

[8] Microcal. <http://www.originlab.com/>.

[9] S. Sekušak, K. R. Liedl, A. Sabljić, J. Phys. Chem. A 102, 1583 (1998).

[10] I. Ljubić, A. Sabljić, J. Phys. Chem. A 106, 4745 (2002).

[11] P. Stoppa, A. Pietropolli Charmet, R. Visinoni, S. Giorgianni, Mol. Phys. 103, 657 (2005).

[12] N. Tasinato, P. Stoppa, A. Pietropolli Charmet, S. Giorgianni, A. Gambi, J. Phys. Chem. A 110, 13412 (2006).

[13] P. Stoppa, A. Pietropolli Charmet, N. Tasinato, S. Giorgianni, A. Gambi, J. Phys. Chem. A 113, 1497 (2009).

[14] K.A. Levenberg, Quart Appl. Math. 2, 164 (1944).

[15] D.V. Marquardt, SIAM J. Appl. Math. 11, 431 (1963). 
[16] K. Madsen, H.B. Nielsen, O. Tingleff, Methods for non-linear least squares problems, $2^{\text {nd }}$ ed. Informatics and mathematical modelling, Technical University of Denmark (2004).

[17] C. Kanzow, N. Yamashita, M. Fukushima, J. Comput. Appl. Math. 172, 375 (2004).

[18] B. Stroustrup, The $\mathrm{C}++$ programming language, $3^{\text {rd }}$ ed. Addison - Wesley (1997).

[19] P.L. Varghese, R.K. Hanson, Appl. Opt. 23, 2376 (1984).

[20] M.A.H. Smith, C.P. Rinsland, B. Fridovich, K.N. Rao, in: K.N. Rao (Ed.), Molecular spectroscopy: Modern research, vol. 3, Academic. Press. Inc., London, pp. 111-248 (1985).

[21] W. J. Thompson, Comput. Phys. 7, 627 (1993).

[22] S.R. Drayson, JQSRT 16, 611 (1976).

[23] M.A. Kuntz, JQSRT 57, 819 (1997).

[24] A.K. Hui, B.H. Armstrong, A.A. Wray, JQSRT 19, 509 (1978).

[25] J. Humliček, JQSRT 21, 309 (1979).

[26] J. Humliček, JQSRT 27, 437 (1982).

[27] K.L. Letchworth, D.C. Benner, JQSRT 107, 173 (2007).

[28] F. Schreier, JQSRT 48, 743 (1992).

[29] R.J. Wells, JQSRT 62, 29 (1999).

[30] S.G. Rautian, I.I. Sobel'man, Sov. Phys. Usp. Engl. Transl. 9 (1967) 701-716.

[31] M. Nelkin, A. Ghatak, Phys. Rev. 135, A4 (1964).

[32] L. Galatry, Phys. Rev. 122, 1218 (1961).

[33] X. Ouyang, P.L. Varghese, Appl. Opt. 28, 1538 (1989). 
[34] L. Joly, V. Zéninari, B. Parvitte, D. Weidmann, D. Courtois, Y. Bonetti, T. Aellen, M. Beck, J. Faist, D. Hofstetter, Appl. Phys. B 77, 703 (2003).

[35] V. Zéninari, L. Joly, B. Grouiez, B. Parvitte, A. Barbe, JQSRT 105, 312 (2007).

[36] R. A. Toth, L. R. Brown, C. Plymate, JQSRT 59, 529 (1998). 
Table 1. List of the dimensionless parameters of the normalized line shape functions.

\begin{tabular}{|c|c|c|}
\hline \multicolumn{2}{|c|}{ Dimensionless Parameter } & \multirow{2}{*}{ Related Dimensional parameters ${ }^{b}$} \\
\hline Definition $^{a}$ & Description & \\
\hline$x=\frac{v-v_{0}}{\alpha_{D}}$ & $\begin{array}{l}\text { Frequency detuning from } \\
\text { resonance frequency }\end{array}$ & $\begin{array}{l}\text { Wavenumber frequency } v \text { and line } \\
\text { centre } v_{0}\left[\mathrm{~cm}^{-1}\right] \text {. }\end{array}$ \\
\hline$y=\frac{\gamma_{L}}{\alpha_{D}}$ & $\begin{array}{l}\text { Frequency of broadening } \\
\text { collisions }\end{array}$ & $\begin{array}{l}\text { Collisional half width, } \gamma_{L} \text {; Pressure } \\
\text { broadening coefficient, } \gamma_{L}^{0}\left[\mathrm{~cm}^{-1} \mathrm{~atm}^{-1}\right]\end{array}$ \\
\hline$z=\frac{\beta}{\alpha_{D}}$ & $\begin{array}{l}\text { Effective frequency of } \\
\text { velocity changing collisions } \\
\text { (weak collision model) }\end{array}$ & $\begin{array}{l}\text { Collisional narrowing, } \beta ; \text { Collisional } \\
\text { narrowing parameter, } \beta^{0}\left[\mathrm{~cm}^{-1} \mathrm{~atm}^{-1}\right] \text {. }\end{array}$ \\
\hline$\zeta=\frac{\Omega}{\alpha_{D}}$ & $\begin{array}{l}\text { Frequency of velocity } \\
\text { changing collisions } \\
\text { (strong collision model) }\end{array}$ & $\begin{array}{l}\text { Collisional narrowing, } \Omega \text {; Collisional } \\
\text { narrowing parameter, } \Omega^{0}\left[\mathrm{~cm}^{-1} \mathrm{~atm}^{-1}\right] .\end{array}$ \\
\hline$s=\frac{\delta}{\alpha_{D}}$ & $\begin{array}{l}\text { Effective frequency of } \\
\text { shifting collision }\end{array}$ & $\begin{array}{l}\text { Line shift, } \delta ; \text { Line shift coefficient, } \delta^{0} \\
{\left[\mathrm{~cm}^{-1} \mathrm{~atm}^{-1}\right] .}\end{array}$ \\
\hline
\end{tabular}

${ }^{a} \alpha_{D}$ denotes the 1/e Doppler half width.

${ }^{b}$ The upper script 0 indicates quantities normalized to a pressure of $1 \mathrm{~atm}$. 
Table 2. Normalized line shape function implemented in VLSFP.

\section{Equation $^{a}$}

\section{Doppler}

Inhomogeneous broadening by random thermal motion.

$$
f(x)=\frac{1}{\sqrt{\pi}} \mathrm{e}^{-x^{2}}
$$

\section{Lorentz}

Homogeneous broadening by internal state perturbing collisions with negligible thermal motion

$$
f(x-s, y)=\frac{1}{\pi} \frac{y}{y^{2}+(x-s)^{2}}
$$

\section{Voigt}

Broadening by thermal motion and internal state perturbing collisions. The two broadening mechanism $f(x-s, y)=\operatorname{Re}[w(x, y)]=\frac{y}{\alpha_{D} \pi^{3 / 2}} \int_{-\infty}^{+\infty} \frac{\mathrm{e}^{-t^{2}}}{[(x-s)-t]^{2}+y^{2}} d t$ are considered to be statistically independent.

\section{Galatry}

Broadening by thermal motion and internal state perturbing collisions; weak collision model for velocity

$$
f(x-s, y, z)=\frac{1}{\pi} \operatorname{Re}\left\{\int_{0}^{+\infty} \mathrm{e}^{-t[y+\mathrm{i}(x-s)]+\left(2 z^{2}\right)^{-1}\left[1-z t-\mathrm{e}^{-z t}\right]} d t\right\}
$$
changing collisions.

\section{Nelkin \& Ghatak / Rautian \&}

\section{Sobel'man}

Broadening by thermal motion and internal state perturbing collisions;

$$
f(x-s, y, \zeta)=\frac{1}{\sqrt{\pi}} \operatorname{Re}\left[\frac{w(x-s, y+\zeta)}{1-\sqrt{\pi \zeta w(x-s, y+\zeta)}}\right]
$$
strong collision model for velocity changing collisions.

\footnotetext{
${ }^{a} w(x, y)$ is the complex probability function.
} 
Table 3. Obtained line parameters of the ro-vibrational transitions of the $v_{1}$ band of $\mathrm{SO}_{2}{ }^{a}$ employed to test VLSFP.

\begin{tabular}{|c|c|c|c|c|c|c|c|c|c|c|}
\hline \multirow{2}{*}{$J^{\prime}$} & \multirow{2}{*}{$K_{a}^{\prime}$} & \multirow{2}{*}{$K_{c}^{\prime}$} & \multirow{2}{*}{$J^{\prime \prime}$} & \multirow{2}{*}{$K_{a}^{\prime \prime}$} & \multirow{2}{*}{$K_{c}^{\prime \prime}$} & \multirow{2}{*}{$\widetilde{v}_{0}\left[\mathbf{c m}^{-1}\right]$} & \multicolumn{2}{|c|}{$\gamma_{\text {self }}^{0}\left[\mathbf{c m}^{-1} \mathbf{a t m}^{-1}\right]$} & \multicolumn{2}{|c|}{$S^{0}\left[10^{-22} \mathrm{~cm} \cdot\right.$ molecule $\left.^{-1}\right]$} \\
\hline & & & & & & & This work & Literature & This work & Literature \\
\hline 20 & 14 & 6 & 21 & 15 & 7 & $1089.52139(7)$ & $0.266(5)$ & $0.26_{4}\left(1_{4}\right)^{b}$ & $5.51(3)$ & $5.67(21)^{b}$ \\
\hline 33 & 11 & 23 & 34 & 12 & 22 & $1089.72440(9)$ & $0.340(10)$ & $0.29_{9}\left(1_{7}\right)^{b}$ & $3.55(6)$ & $2.99(18)^{b}$ \\
\hline 29 & 18 & 12 & 29 & 19 & 11 & $1089.79423(13)$ & $0.20(2)$ & $0.21_{7}\left(1_{1}\right)^{c}$ & $0.65(2)$ & $0.746(101)^{d}$ \\
\hline 51 & 7 & 45 & 52 & 8 & 44 & $1089.8234(2)$ & $0.40(6)$ & - & $0.51(5)$ & - \\
\hline 24 & 13 & 11 & 25 & 14 & 12 & $1089.84713(3)$ & $0.268(7)$ & $0.28_{3}\left(1_{3}\right)^{c}$ & $5.70(5)$ & $5.66(11)^{d}$ \\
\hline 15 & 15 & 1 & 16 & 16 & 0 & $1089.87257(4)$ & $0.161(7)$ & $0.19_{2}\left(1_{0}\right)^{c}$ & $6.78(8)$ & $6.91(12)^{d}$ \\
\hline 32 & 10 & 22 & 33 & 11 & 23 & $1089.7409(2)^{e}$ & $0.27(4)$ & $0.25_{0}\left(2_{1}\right)^{c}$ & $0.71(4)$ & $0.563(12)^{d}$ \\
\hline
\end{tabular}

${ }^{a}$ Figures in parentheses are one standard deviations.

${ }^{b}$ From Ref. [34].

${ }^{\mathrm{c}}$ From Ref. [35]; the quoted uncertainties have been derived from the error bars of Figures 4, 6 and 8.

${ }^{d}$ From Ref. [35].

${ }^{e}$ Belonging to $v_{1}+v_{2}-v_{2}$ of ${ }^{32} \mathrm{SO}_{2}$; an overlapping with $16_{13,3} \leftarrow 17_{14,4}$ of ${ }^{34} \mathrm{SO}_{2} v_{1}$ band also occurs. 
Table 4. Obtained line parameters of the ro-vibrational transitions of the $v_{7}$ band of $\mathrm{CH}_{2} \mathrm{CHF}^{a}$.

\begin{tabular}{|c|c|c|c|c|c|c|c|c|c|}
\hline \multirow{2}{*}{$J^{\prime}$} & \multirow{2}{*}{$K_{a}^{\prime}$} & \multirow{2}{*}{$K_{c}^{\prime}$} & \multirow{2}{*}{$J^{\prime \prime}$} & \multirow{2}{*}{$K_{a}^{\prime \prime}$} & \multirow{2}{*}{$K_{c}^{\prime \prime}$} & \multirow{2}{*}{$\tilde{\mathbf{v}}_{0}\left[\mathrm{~cm}^{-1}\right]$} & \multicolumn{2}{|c|}{$\gamma_{\text {self }}^{0}\left[\mathbf{c m}^{-1} \mathbf{a t m}^{-1}\right]$} & \multirow{2}{*}{$S^{0}\left[10^{-21} \mathrm{~cm} \cdot \mathrm{molecule}^{-1}\right]$} \\
\hline & & & & & & & Voigt & Nelkin - Ghatak & \\
\hline 10 & 1 & 10 & 11 & 1 & 11 & $1148.1499(8)$ & $0.80(10)$ & $0.78(2)$ & $2.8(2)$ \\
\hline 10 & 7 & 3 & 11 & 7 & 4 & $1148.1963(4)$ & - & $0.69(6)$ & $3.05(4)$ \\
\hline 10 & 8 & 2 & 11 & 8 & 3 & $1148.30268(13)$ & $0.48(4)$ & $0.88(4)$ & $2.88(2)$ \\
\hline 9 & 1 & 8 & 10 & 1 & 9 & $1148.3454(2)$ & $0.74(2)$ & $0.80(2)$ & $4.253(13)$ \\
\hline 8 & 0 & 8 & 9 & 1 & 9 & $1148.3689(2)$ & - & - & $0.72(2)$ \\
\hline 10 & 9 & 1 & 11 & 9 & 2 & $1148.4210(2)$ & $0.31(2)$ & $0.54(6)$ & $2.59(2)$ \\
\hline 9 & 2 & 7 & 10 & 2 & 8 & $1148.4327(2)$ & $0.75(2)$ & $0.39(3)$ & $4.29(2)$ \\
\hline 9 & 1 & 8 & 9 & 2 & 7 & $1148.4574(4)$ & $0.51(5)$ & $0.87(10)$ & $0.63(3)$ \\
\hline 20 & 1 & 19 & 20 & 2 & 18 & $1148.5396(3)$ & - & - & $0.70(4)$ \\
\hline 10 & 10 & 0 & 11 & 10 & 1 & $1148.5513(3)$ & - & - & $0.85(2)$ \\
\hline 9 & 3 & 6 & 10 & 3 & 7 & $1148.5644(3)$ & $0.47(6)$ & $0.45(5)$ & $3.30(5)$ \\
\hline 9 & 3 & 7 & 10 & 3 & 8 & $1148.5742(3)$ & - & $0.65(10)$ & $4.6(2)$ \\
\hline 9 & 2 & 8 & 10 & 2 & 9 & $1148.5792(3)$ & - & $0.84(6)$ & $5.3(3)$ \\
\hline
\end{tabular}

${ }^{a}$ Figures in parentheses are one standard deviations. 


\section{Figure Captions}

Figure 1. Block diagram representing the implementation of VLSFP. Each box represents a class referred to as Object (C. M. stands for collision model). The objects interact with each other to make the application: the arrow means "derives from", the diamond means "makes use of" (see text for discussion).

Figure 2. A single line fit obtained employing VLSFP. Row (a) shows the experimental $(\bigcirc)$ and computed spectrum (-). Experimental details: $\mathrm{SO}_{2}$ total pressure $=107.0 \mathrm{~Pa}$, path length $=92.3 \mathrm{~cm}$, temperature $=297$ K. The remaining rows show the residuals obtained from different models: (b) Voigt; (c) weak collision model (wcm); (d) strong collision model $(\mathrm{scm})$. The standard deviations of the fits, $\sigma\left(\mathrm{cm}^{-1}\right)$, are: $\sigma_{\text {Voigt }}=$ $1.6 \times 10^{-3} ; \sigma_{\mathrm{wcm}}=0.88 \times 10^{-3} ; \sigma_{\mathrm{scm}}=0.90 \times 10^{-3}$.

Figure 3. Multi - line fit of the $\mathrm{SO}_{2}$ spectrum between 1089.69 and $1089.87 \mathrm{~cm}^{-1}$ : row (a) shows the experimental $(\bigcirc)$ and computed spectrum $(-)$. Experimental details: $\mathrm{SO}_{2}$ total pressure $=511.4 \mathrm{~Pa}$, path length $=92.3$ $\mathrm{cm}$, temperature $=297 \mathrm{~K}$. The remaining rows show the residuals obtained from different models: (b) Voigt; (c) weak collision model (wcm); (d) strong collision model ( $\mathrm{scm}$ ). The standard deviations of the fits, $\sigma\left(\mathrm{cm}^{-1}\right)$, are: $\sigma_{\mathrm{Voigt}}=4.0 \times 10^{-3} ; \sigma_{\mathrm{wcm}}=3.1 \times 10^{-3} ; \sigma_{\mathrm{scm}}=3.2 \times 10^{-3}$. 
Figure 4. Comparison between the determined line parameters $(\nabla)$ and the values of Refs. [34] $(\diamond)$ and [35] ( $\bigcirc)$. Upper trace: self broadening coefficients; lower trace: line strengths.

Figure 5. Vinyl fluoride spectrum between 1148.14 and $1148.6 \mathrm{~cm}^{-1}$ : (a) experimental $(\bigcirc)$ and computed spectrum $(-)$. Experimental details: $\mathrm{CH}_{2} \mathrm{CHF}$ total pressure $=30.6 \mathrm{~Pa}$, path length $=92.3 \mathrm{~cm}$, temperature $=$ $299 \mathrm{~K}$. The remaining rows show the residuals obtained from different models: (b) Voigt; (c) strong collision model (scm); The standard deviations of the fits, $\sigma\left(\mathrm{cm}^{-1}\right)$, are: $\sigma_{\text {Voigt }}=4.5 \times 10^{-3} ; \sigma_{\mathrm{scm}}=2.6 \times 10^{-3}$. 


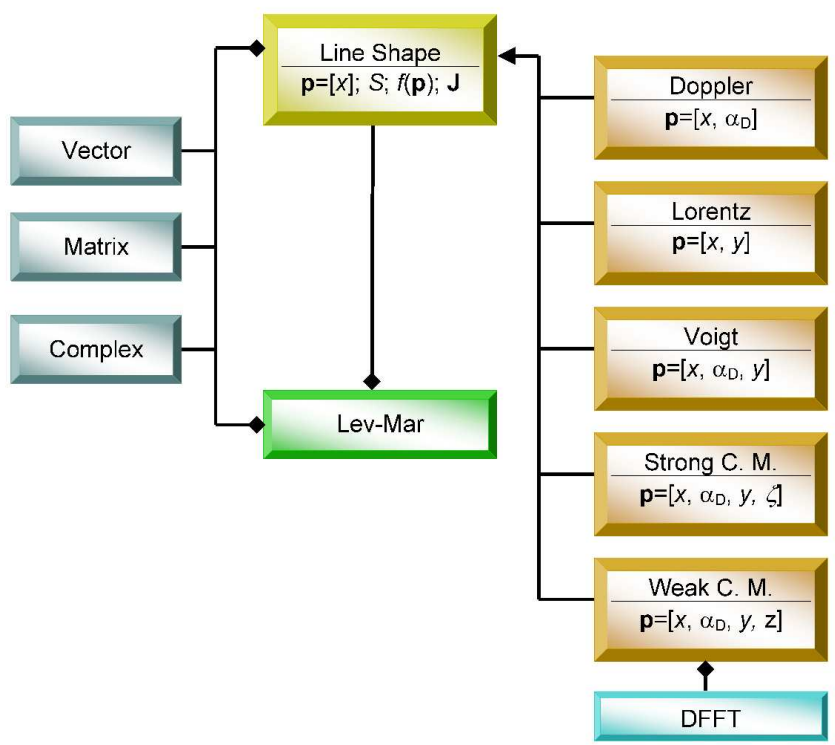

Figure 1

$297 \times 210 \mathrm{~mm}(600 \times 600 \mathrm{DPI})$

URL: http://mc.manuscriptcentral.com/tandf/tmph 


1
2
3
4
5
6
7
8
9
10
11
12
13
14
15
16
17
18
19
20
21
22
23
24
25
26
27
28
29
30
31
32
33
34
35
36
37
38
39
40
41
42
43
44
45
46
47
48
49
50
51
52
53
54
55
56
57
59
60

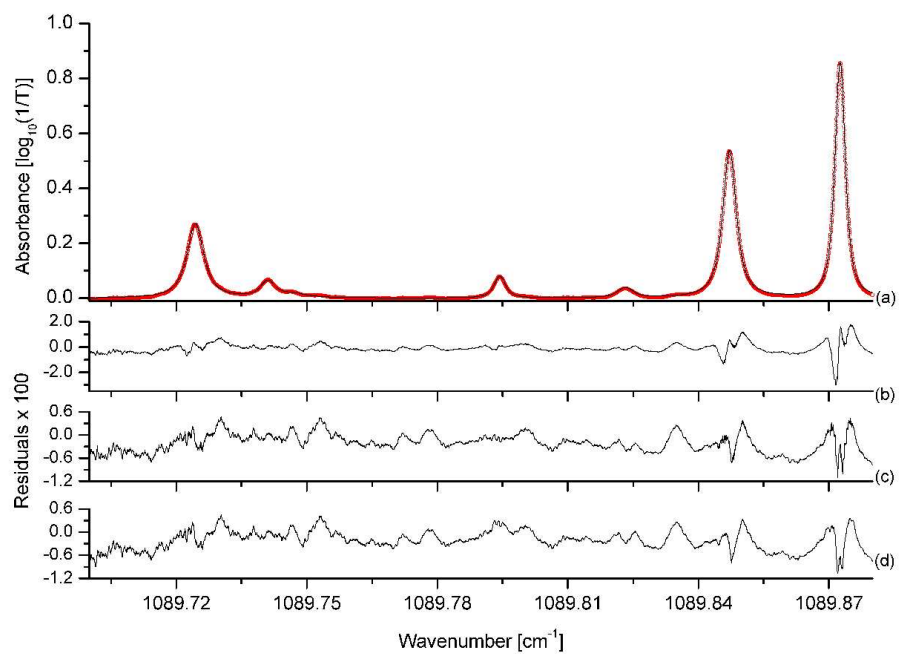

Figure 3

$297 \times 210 \mathrm{~mm}(600 \times 600 \mathrm{DPI})$

URL: http://mc.manuscriptcentral.com/tandf/tmph 


1
2
3
4
5
6
7
8
9
10
11
12
13
14
15
16
17
18
19
20
21
22
23
24
25
26
27
28
29
30
31
32
33
34
35
36
37
38
39
40
41
42
43
40
45
49
50
51
52
53
55
50

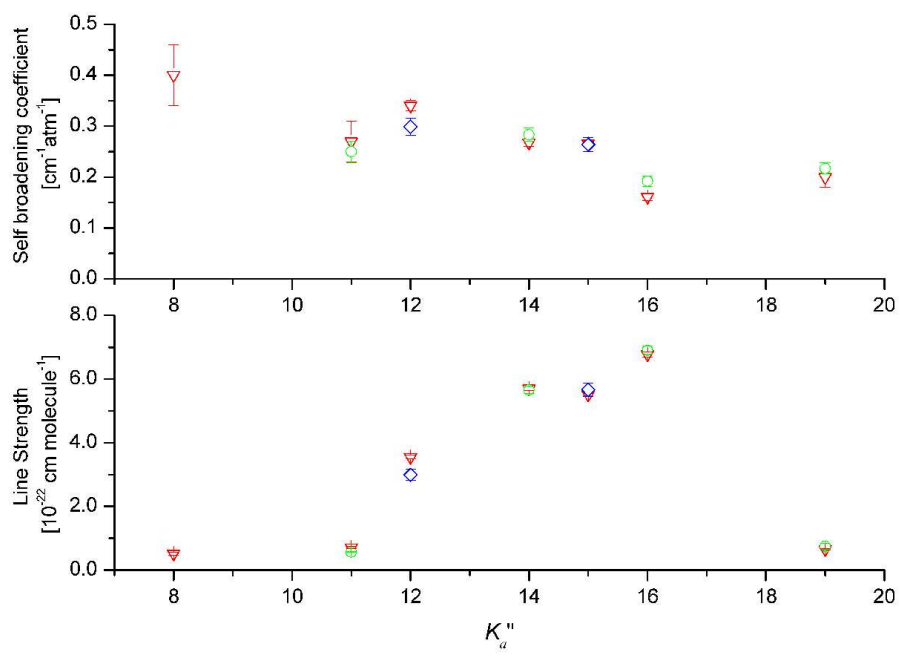

Figure 4

$297 \times 210 \mathrm{~mm}(600 \times 600 \mathrm{DPI})$

URL: http://mc.manuscriptcentral.com/tandf/tmph 


1
2
3
4
5
6
7
8
9
10
11
12
13
14
15
16
17
18
19
20
21
22
23
24
25
26
27
28
29
30
31
32
33
34
35
36
37
38
39
40
41
42
43
44
45
46
47
48
49
50
51
52
53
54
55
56
57
59
60

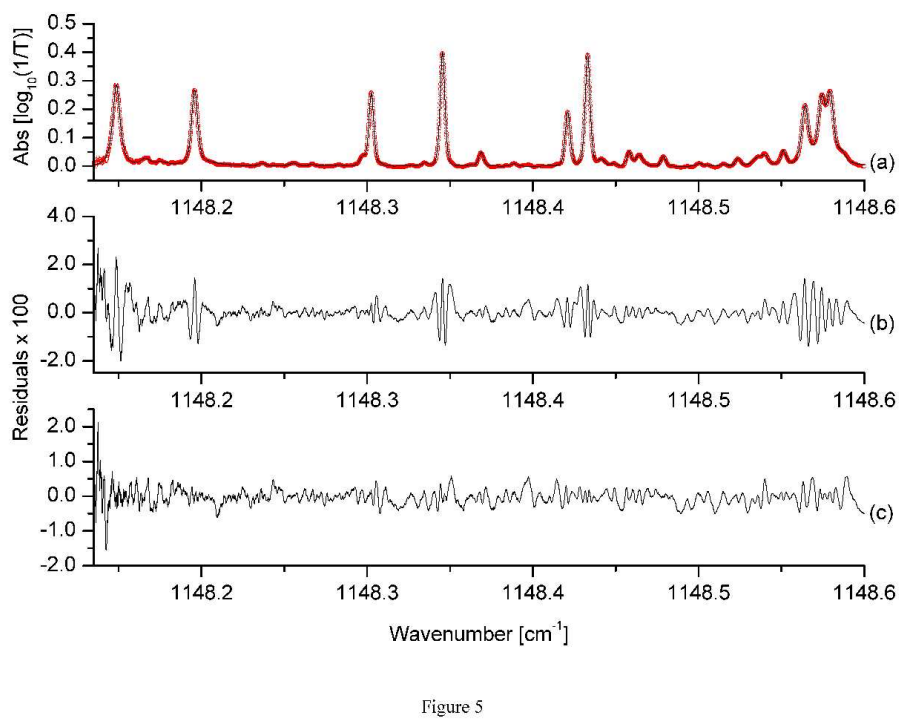

$297 \times 210 \mathrm{~mm}(600 \times 600 \mathrm{DPI})$

URL: http://mc.manuscriptcentral.com/tandf/tmph 


\title{
Determination of the Vinyl Fluoride line intensities by TDL spectroscopy: the Object Oriented approach of Visual Line Shape Fitting Program to the line profile analysis
}

\author{
Nicola Tasinato*, Andrea Pietropolli Charmet, Paolo Stoppa, Santi Giorgianni
}

Università Ca' Foscari di Venezia - DCF - I-30123 Venezia (Italy)

\begin{abstract}
In this work the self-broadening coefficients and the integrated line intensities for a number of ro-vibrational transitions of vinyl fluoride have been determined for the first time by means of TDL spectroscopy. The spectra recorded in the atmospheric window around $8.7 \mu \mathrm{m}$ appear very crowded with a density of about 90 lines per $\mathrm{cm}^{-1}$. In order to fit these spectral features a new fitting software has been implemented. The program which is designed for laser spectroscopy, can fit many lines simultaneously on the basis of different theoretical profiles (Doppler, Lorentz, Voigt, Galatry and Nelkin Ghatak). Details of the Object Oriented implementation of the application are given. The reliability of the program is demonstrated by determining the line parameters of some ro-vibrational lines of sulphur dioxide in the $v_{1}$ band region around $9 \mu \mathrm{m}$. Then the software is used for the line profile analysis of vinyl fluoride. The experimental line shapes show deviations from the Voigt profile which can be well modelled by using a Dicke narrowed line shape function. This leads to the determination of the selfnarrowing coefficient within the framework of the strong collision model.
\end{abstract}

Keywords: Vinyl fluoride; Line parameter determination; Line shape fitting software; Weak and strong collision models; Voigt and Dicke narrowed profiles.

\footnotetext{
* Corresponding author.

E-mail: tasinato@unive.it; Phone: +39 041 2348598; Fax: +39 0412348594

Address: Università Ca' Foscari Venezia, Dipartimento di Chimica Fisica, Dorsoduro 2137, I-30123 VENEZIA (Italy)
} 


\section{Introduction}

The study of the Earth and planetary atmospheres and of the interstellar medium by means of spectroscopic techniques has rapidly grown up since the earlier experiments in the second half of the 1900. Among the various techniques, infrared spectroscopy plays a significant role, in particular for remote sensing of the terrestrial atmosphere. In fact, IR remote sensing permits to monitor and accurately retrieve the concentration of the atmospheric constituents and trace pollutants. Though, in order to obtain reliable measurements, an accurate knowledge of the line parameters, such as line centres, intensities and pressure broadening coefficients, is required.

Thanks to the availability of always more powerful computers, the time consuming task of inverting the experimental spectra to extract spectroscopic parameters has become increasingly assisted by computer programs. Compared to the huge number of software available for extracting the Hamiltonian's parameters from the assigned line positions (a list of freely available devices is given in [1]), some programs specifically designed to perform the line shape analysis by fitting the experimental spectrum have been presented (see, for example, $[2-6]$ ). It is worthwhile to note that these programs appear specifically designed to deal mostly with FTIR spectra. As a consequence, during the fitting procedure, they explicitly take into account the step of the parameterization of the FTIR instrumental line shape. These software, based on the multi-spectrum fitting technique, are the best choice when the large number of lines recorded by the interferometer in a single scan needs to be analyzed.

On the other hand, the spectrometers based on laser sources have a simpler instrumental contribution, generally modelled as a Gaussian or Lorentzian function. 
Furthermore, the spectral region which can be recorded in a scan is of the order of some wavenumbers, thus greatly reducing the amount of lines to be accounted for in a single spectrum. In many cases, the line shape analysis is carried out by using home made programs which are sometimes designed to fit the experimental features to a single theoretical function. Further, some programs are able to fit only one absorption line at time and still run from a command line window, thus avoiding an immediate comparison between the experimental data and the theoretical model. Commercial packages, such as IGOR Pro [7] and Origin [8], can be employed as well, although they are not completely well suited for this purpose.

Vinyl Fluoride, $\mathrm{CH}_{2} \mathrm{CHF}$, is widely used by industry mainly as monomer for the production of synthetic materials such as poly-vinyl fluoride. The potential role of halogenated alkenes as air pollutants has motivated a number of spectroscopic investigations and studies about their reactivity toward hydroxyl radical and ozone (see Refs. $[9,10]$ and references therein).

The infrared spectra of vinyl fluoride have been deeply investigated by our research group (see Refs. [11 - 13] and references therein). The studies have mainly dealt with the analysis of the high resolution spectra and the obtained results have led to the determination of accurate spectroscopic parameters for a number of fundamental and overtone vibrations and a thorough understanding of the observed perturbations. Recently, the medium resolution spectrum has been studied in the $400-8000 \mathrm{~cm}^{-1}$ region and the integrated absorption cross sections have been determined for the first time [13]. In addition, ab-initio calculations with large basis sets have been performed at the $\operatorname{CCSD}(\mathrm{T})$ level of theory leading to the determination of harmonic and anharmonic force fields. 
Despite the industrial importance of vinyl fluoride, there exist no studies on the determination of the line parameters of the ro-vibrational absorption lines. The lack of literature data is partly motivated by the complex structure of the high resolution spectrum. In the present work we report the very first results obtained from the line shape analysis in the $v_{7}$ band region around $1148 \mathrm{~cm}^{-1}$ by means of tunable diode laser (TDL) spectroscopy. In this region, the spectrum of vinyl fluoride appears very crowded even at low pressures. Hence, the analysis strongly requires a fitting software capable to simultaneously fit all the absorptions present in the spectral micro-windows acquired by the TDL system. For this reason we implemented a new software for the line shape fitting and the retrieval of the line parameters. The program, called Visual Line Shape Fitting Program (VLSFP), can fit several lines at once using different theoretical line shape functions. These include the Doppler, Lorentz and Voigt profiles, as well as the Galatry and Nelkin - Ghatak functions. The reliability of VLSFP has been tested by retrieving the sulphur dioxide line parameters and comparing them with the literature values. In this paper we also describe the program implementation and validation. In Section 2 the adopted experimental procedure is outlined while Sections 3 and 4 are concerned with the description of the fitting software and its test. Then in Section 5 we illustrate the results of the line shape analysis of vinyl fluoride. Conclusions and future work are addressed in Section 6.

\section{Experimental procedure and data inversion}

The high resolution spectra of either sulphur dioxide or vinyl fluoride were acquired on our tunable diode laser spectrometer employing a $92.3( \pm 0.2) \mathrm{cm}$ path 
length cell with $\mathrm{KBr}$ windows. The gas sample pressures were measured employing an Alcatel ARD 1003 capacitance gauge with a full scale range of $1000 \mathrm{~Pa}$ and a quoted manufacturer's accuracy of 1.5 Pa. Before each measurements the cell was evacuated down to about $10^{-4} \mathrm{~Pa}$; an elapse time of 10 and 20 minutes, for $\mathrm{SO}_{2}$ and $\mathrm{CH}_{2} \mathrm{CHF}$ respectively, between the filling of the cell and the spectrum acquisition was adopted. The spectra were calibrated employing the $\mathrm{SO}_{2}$ line frequencies obtained by high resolution FTIR measurements. The $\mathrm{SO}_{2}$ and $\mathrm{CH}_{2} \mathrm{CHF}$ gas samples were provided by Sigma - Aldrich (99.9\% purity) and Peninsular Chemical Research Inc. (99\% purity), respectively, and were used without any further purification; no buffer gasses were employed. The $\mathrm{SO}_{2}$ spectra were acquired at $297( \pm 1) \mathrm{K}$ and the pressures were varied in the range between 15 and $800 \mathrm{~Pa}$. The measurements on $\mathrm{CH}_{2} \mathrm{CHF}$ were carried out at $299( \pm 1) \mathrm{K}$ with pressures ranging from 7 to $68 \mathrm{~Pa}$.

Since the instrumental response of our TDL spectrometer is well described by a Gaussian function, its contribution to the line shapes was explicitly taken into account during the fitting procedure by fixing the Doppler half width to an effective value, $\gamma_{D}^{\text {eff }}$, given by

$$
\gamma_{D}^{\text {eff }}=\sqrt{\left(\gamma_{D}^{\text {sample }}\right)^{2}+\left(\gamma_{D}^{T D L}\right)^{2}}
$$

where $\gamma_{D}^{\text {sample }}$ and $\gamma_{D}^{T D L}$ are the molecular and instrumental Doppler half widths, respectively. The value of $\gamma_{D}^{\text {eff }}$ was retrieved by fitting the spectral lines recorded at low pressure (less than $30 \mathrm{~Pa}$ and $10 \mathrm{~Pa}$ for $\mathrm{SO}_{2}$ and $\mathrm{CH}_{2} \mathrm{CHF}$, respectively) to a Gaussian profile. 


\section{Visual Line Shape Fitting Program implementation: an Object Oriented approach}

VLSFP has been written combining Microsoft Visual Basic and C\# for the graphical interface and the computational kernel, respectively. The determination of the line parameters within a given theoretical model is performed through an iterative procedure using the Levenberg - Marquardt algorithm $[14,15]$. The algorithm has been implemented following the pseudo-code described in [16] and then modified in order to set bounds to the variability of the parameters or to keep the parameter values constrained, as described by Kanzow et al. [17]. In the current implementation, the algorithm terminates when the maximum number of iterations is completed or when one of the convergence criteria is met. These criteria are related to the square of the Euclidean norm of the residuals, its gradient and to the variation of the parameters between two subsequent iterations. The fitting routine runs as a background process avoiding the program interface to be frozen during the execution of the fitting task.

The computational kernel, schematically depicted in Figure 1, has been designed following an Object Oriented Programming approach [18]. This programming technique, born around 1960s, uses objects and their interactions to design computer programs. An application is then composed of a collection of cooperating objects, rather than an ensemble of subroutines that compute specific tasks. Each box of Figure 1 is referred to as class and it represents an "object". The class defines the properties (the class variables) of the corresponding object and its behaviour (the class methods). The object is able to process data and mostly to interact with other objects by receiving and sending messages. In a pictorial representation, every single object can be seen as a little 
machine with its own task. The set of distinct machines then cooperate to accomplish an objective: in our case the fit of the experimental line shape by means of different theoretical profiles.

Referring to Figure 1, the classes Vector, Matrix and Complex implement vector, matrix and complex algebra, respectively. They are used by the other objects of the project to store and process the data. The matrices have been implemented as onedimensional arrays thus speeding up the access to their elements. The fitting routines have been encapsulated inside the class LevMar and they have been designed to deal with a generic object of the type Line Shape. Line Shape represents an abstract class, that is an abstract object which defines the common characteristics possessed by all the line shapes (for example, the computed line profile, the line parameters array and the Jacobian matrix). The single line shape functions have been derived from the main abstract class and within each class a specific implementation of the methods has been made. As a consequence, new line shape functions, such as the speed dependent profiles, can be implemented without changing neither the LineShape nor the LevMar classes.

The line shape functions have been implemented in the normalized form. Denoting the generic line function by $f(\mathbf{p})$, it is normalized to unitary area:

$$
\int_{-\infty}^{+\infty} f(\mathbf{p}) d x=1
$$

where $\mathbf{p}=[x, y, z, \ldots]$ is the vector of dimensionless parameters which are described in Table 1 following the notation of Varghese and Hanson [19]. As it can be seen, the line parameters are normalized by the effective Doppler half width defined as 


$$
\alpha_{D}=\frac{\gamma_{D}}{\sqrt{\ln 2}}
$$

where $\gamma_{D}$ is the dimensional Doppler half width given by [20]

$$
\gamma_{D}=\frac{v_{0}}{c}\left(\frac{2 k_{\mathrm{B}} N_{\mathrm{A}} T \ln 2}{m}\right)^{1 / 2}
$$

where $v_{0}$ is the resonant frequency; $c$ is the speed of light; $k_{\mathrm{B}}$ is the Boltzman's constant; $N_{\mathrm{A}}$ is the Avogadro's number; $T(\mathrm{~K})$ is the absolute temperature, $m$ (a. m. u.) is the mass of the absorbing gas.

According to Beer - Lambert's law, the transmission of monochromatic radiation travelling a path of length $\ell$ through a uniform gas at pressure $p$ is given by

$$
I(v)=I_{0} \mathrm{e}^{-k(v) p \ell}
$$

where $I_{0}$ is the unattenuated intensity of the radiation having frequency $v$. The quantity $k(v)$ is called the absorption coefficient and, for a single absorption line, it can be expressed in the form:

$$
k(v)=S f\left(v-v_{0}\right)
$$


where $S$ is the integrated absorption coefficient (also referred to as line intensity) and $f\left(v-v_{0}\right)$ is the line shape function as defined in equation (2). The dimensionless line shape functions implemented in VLSFP are listed in Table 2. During the fitting procedure, VLSFP refines the line intensity $S$, the line frequency centre $v_{0}$ and the remaining line parameters, which clearly depend on the specific line shape function.

The Voigt profile is widely used for modelling the spectral line profiles and in other scientific areas ([21] and references therein), and hence the issue of its computation has been dealt over long time and intensive efforts have led to the development of many algorithms [22 - 27]. A comprehensive survey of the different computational approaches has been reported by Schreier [28]. In VLSFP, the Voigt function is computed employing the optimized version of Humliček's code proposed by Wells [29]. Since this algorithm computes also the imaginary part of the complex probability function, it can be conveniently used for the computation of the Nelkin Ghatak profile [30, 31], as well.

Concerning the Galatry function [32], the profile is computed by the Fourier transform of the corresponding correlation function, $\Phi(y, z, t)$, following the algorithm proposed by Ouyang and Varghese [33]:

$$
f(x-s, y, z)=\frac{1}{\pi} \operatorname{Re}\{\Im[\Phi(y, z, t)]\}
$$

where $\mathfrak{I}$ indicates the Fourier transform operator. The derivatives are in turn computed via the Fourier transform of the appropriate correlation function. As a consequence, a discrete fast Fourier transform routine (DFFT) has been implemented. 


\section{Test of VLSFP with $\mathrm{SO}_{2}$ lines}

VLSFP has been tested by retrieving the parameters of some ro-vibrational lines of sulphur dioxide in the region around $9 \mu \mathrm{m}$. This region is characterized by the absorptions of the $v_{1}$ fundamental corresponding to the symmetric stretching normal mode. In this section the results for a number of lines are presented in order to demonstrate the reliability of the application.

An example of the fits obtained for an isolated absorption line is given in Figure 2. When fitted to a Voigt profile, this line seems to show the effect of Dicke narrowing as suggested by the shape of the residuals. As a consequence, the line has also been fitted employing the weak and strong collision models. As can been seen from the plots of the residuals, the models accounting for the velocity changing collision effects reproduce properly the observed line shape.

A wider spectral region is shown in Figure 3. In this case, the lines have been fitted to a Voigt profile, employing the multi - line fitting procedure. In general, all the lines have been well reproduced although the residuals show some regular trends. This behaviour can be partly explained by the presence of very weak lines underneath the stronger ones. These lines, being not resolved at all, can not be taken into account during the fits but at the same time cause asymmetries in the observed absorptions. For the same reason, the Dicke narrowed profiles are not able to account for all the deviations. Anyway, even in such situation, the physical meaning of the parameters is preserved. This has been confirmed by plotting the collisional half widths and the line intensities as a function of the gas pressure: the line parameters lie on straight lines passing through the origin. 
The obtained line parameters are listed in Table 3 together with the more recent literature values $[34,35]$ whose comparison is shown in Figure 4. As it can be seen, the present data and the literature values agree in a very satisfactory manner, indicating the reliability of the retrieved line parameters and hence validating the computational kernel of VLSFP.

\section{Determination vinyl fluoride line shape parameters}

The spectrum of vinyl fluoride in the $8.7 \mu \mathrm{m}$ atmospheric region is characterized by the ro-vibrational transitions belonging to the $v_{7}$ band which corresponds to the $\mathrm{C}-\mathrm{F}$ stretching. As pointed out in the introduction, this spectral region is very crowded: the high density of lines, about 90 lines per $\mathrm{cm}^{-1}$, can be appreciated in Figure 5 (a). Besides the stronger absorption of the $v_{7}$ normal mode, the majority of the weakest lines is due to the ro-vibrational transitions of the $v_{7}+v_{9}-v_{9}$ hot band. Due to their intensity these lines strongly affect the main features and therefore they must be accounted during the fitting procedure.

When the spectral lines are fitted to the Voigt profile, the shape of the residuals shows significant deviations from this model, as illustrated in Figure 5 (b). For this reason the lines have also been fitted employing the Nelkin - Ghatak profile. By using this strong collision model there is a dramatic improvement of the residuals as it is shown in Figure 5 (c): the standard deviation of the fit decreases by about a factor of two. Therefore, in the pressure range investigated the self-broadened vinyl fluoride sample appears as system strongly affected by the motional narrowing effect in which 
either the velocity changing collisions and the internal state - dephasing collisions play an almost equal role in determining the shape of the spectral lines. Consequently, the line profiles accounting for the Dicke narrowing effect must be employed to properly model the experimental features.

The retrieved line parameters for the $v_{7}$ lines are listed in Table 4. The line parameters of the hot band have been determined as well, but they are not given here because of the lack of a reliable assignment of the ro-vibrational transitions at present. Again, we preferred to not report the self-broadening coefficient of three lines because their accuracy is too low. This is due to the small range of vinyl fluoride pressures adopted in the experiments which limits the amount of pressure broadening for some of the lines. On the other hand, the use of low pressures is strongly recommended in order to avoid the saturation of the absorptions, and hence working in the experimental conditions of applicability of the Beer - Lambert law. As can be seen from Table 4, the obtained self-broadening coefficients ranges from 0.4 to $0.9 \mathrm{~cm}^{-1} \mathrm{~atm}^{-1}$. Such a wide interval is shown, for instance, by water vapour, whose self-broadening parameters are between 0.1 and $0.5 \mathrm{~cm}^{-1} \mathrm{~atm}^{-1}$ [36]. Besides, to a first investigation no dependence on the quantum numbers have been observed. This is understandable by considering the reduced set of data in which the majority of lines belong to the ${ }^{\mathrm{Q}} \mathrm{P}$ subband. Therefore, in order to draw a more reliable conclusion about the quantum number dependence of the self-broadening coefficients, the analysis need to extended to a spectral region as wide as possible including the B-type transitions, as well.

The fits employing the Nelkin - Ghatak profile led to the determination of the narrowing parameter which result to be $0.40( \pm 0.08) \mathrm{cm}^{-1} \mathrm{~atm}^{-1}$. As can be seen, the magnitude of the self-broadening and self-narrowing have a similar extent. 


\section{Conclusions and Outlook}

The very first results of the line shape analysis of the vinyl fluoride infrared absorptions have been presented and discussed. In addition, the details of the implementation and validation of the fitting software (VLSFP) used for the analysis have been given.

The program has been specifically designed to deal with the spectra recorded by means of laser based spectrometers in which the instrumental function can be modelled as a Gaussian or Lorentzian functions. Besides these two functions, the spectral absorptions can be fitted employing the Voigt profile as well as the weak and strong collision models which account for the Dicke narrowing effect. The application has been implemented following a strongly Object Oriented Programming approach which leads to an easier maintenance and future developments. The program runs under Windows environment and it has a standard graphical interface according to the version of the operation system currently in use. In order to test the program, the line parameters of some sulphur dioxide lines have been determined and compared with the literature data. Once validated, VLSFP has been used to obtain the line parameters of $\mathrm{CH}_{2} \mathrm{CHF}$.

The vinyl fluoride spectra in the $1148 \mathrm{~cm}^{-1}$ atmospheric region have been recorded with our TDL spectrometer at $299 \mathrm{~K}$. The measurements have been carried out on a self-broadened $\mathrm{CH}_{2} \mathrm{CHF}$ sample with pressures in the range between 7 and $68 \mathrm{~Pa}$ : even at these low gas pressures the spectrum presents a high density of absorption lines. The self-broadening and integrated absorption coefficients for 13 lines belonging to the $\mathrm{R}$ branch of the $v_{7}$ normal mode have been determined for the first time. The experimental line shapes showing deviations from the Voigt profile have also been 
fitted using the Nelkin - Ghatak profile. This has led to the determination of the self narrowing coefficient.

The present paper gives an overview of the work that we are carrying out at present. Indeed, on the basis of the good results obtained from the analysis of the vinyl fluoride crowded spectra, we are going to extend the investigation in a spectral region as wide as possible with the aim to derive the vinyl fluoride line parameters in the $8-9$ $\mu \mathrm{m}$ atmospheric window. Future work will also be devoted toward a definitive assignment of the transitions belonging to the $v_{7}+v_{9}-v_{9}$ hot band and to an improvement of the accuracy for some of the broadening coefficients.

\section{Acknowledgments}

The authors thank Prof. P. L. Varghese for the code for the computation of the Galatry function. The authors also gratefully acknowledge Prof. J. M. Flaud for the $\mathrm{SO}_{2}$ data about $v_{1}$ and $v_{1}+v_{2}-v_{2}$ upon which the assignments have been made. This work was financially supported by PRIN 2007 (project: "Trasferimenti di energia, carica e molecole in sistemi complessi”). 


\section{References}

[1] Z. Kisiel, PROSPE - Programs for Rotational SPEctroscopy, <http:// info.ifpan.edu.pl/ kisiel/prospe.htm/>.

[2] M. Carlotti, Appl. Opt. 27, 3250 (1988).

[3] D.C. Benner, C.P. Rinsland, V.M. Devi, M.A.H. Smith, D.A. Atkins, JQSRT 53, 705 (1995).

[4] A.S. Pine, T. Gabard, JQSRT 66, 69 (2000).

[5] D. Jacquemart, J.Y. Mandin, V. Dana, N. Picqué, G.A. Guelachvili, Eur. Phys. J. D 14, 55 (2001).

[6] J.J. Plateaux, L. Ragalia, C. Boussin, A. Barbe, JQSRT 68, 507 (2001).

[7] Wavemetrics Inc. <http://www.wavemetrics.com>.

[8] Microcal. <http://www.originlab.com/>.

[9] S. Sekušak, K. R. Liedl, A. Sabljić, J. Phys. Chem. A 102, 1583 (1998).

[10] I. Ljubić, A. Sabljić, J. Phys. Chem. A 106, 4745 (2002).

[11] P. Stoppa, A. Pietropolli Charmet, R. Visinoni, S. Giorgianni, Mol. Phys. 103, 657 (2005).

[12] N. Tasinato, P. Stoppa, A. Pietropolli Charmet, S. Giorgianni, A. Gambi, J. Phys. Chem. A 110, 13412 (2006).

[13] P. Stoppa, A. Pietropolli Charmet, N. Tasinato, S. Giorgianni, A. Gambi, J. Phys. Chem. A 113, 1497 (2009).

[14] K.A. Levenberg, Quart Appl. Math. 2, 164 (1944).

[15] D.V. Marquardt, SIAM J. Appl. Math. 11, 431 (1963). 
[16] K. Madsen, H.B. Nielsen, O. Tingleff, Methods for non-linear least squares problems, $2^{\text {nd }}$ ed. Informatics and mathematical modelling, Technical University of Denmark (2004).

[17] C. Kanzow, N. Yamashita, M. Fukushima, J. Comput. Appl. Math. 172, 375 (2004).

[18] B. Stroustrup, The C++ programming language, $3^{\text {rd }}$ ed. Addison - Wesley (1997).

[19] P.L. Varghese, R.K. Hanson, Appl. Opt. 23, 2376 (1984).

[20] M.A.H. Smith, C.P. Rinsland, B. Fridovich, K.N. Rao, in: K.N. Rao (Ed.), Molecular spectroscopy: Modern research, vol. 3, Academic. Press. Inc., London, pp. 111-248 (1985).

[21] W. J. Thompson, Comput. Phys. 7, 627 (1993).

[22] S.R. Drayson, JQSRT 16, 611 (1976).

[23] M.A. Kuntz, JQSRT 57, 819 (1997).

[24] A.K. Hui, B.H. Armstrong, A.A. Wray, JQSRT 19, 509 (1978).

[25] J. Humliček, JQSRT 21, 309 (1979).

[26] J. Humliček, JQSRT 27, 437 (1982).

[27] K.L. Letchworth, D.C. Benner, JQSRT 107, 173 (2007).

[28] F. Schreier, JQSRT 48, 743 (1992).

[29] R.J. Wells, JQSRT 62, 29 (1999).

[30] S.G. Rautian, I.I. Sobel'man, Sov. Phys. Usp. Engl. Transl. 9 (1967) 701-716.

[31] M. Nelkin, A. Ghatak, Phys. Rev. 135, A4 (1964).

[32] L. Galatry, Phys. Rev. 122, 1218 (1961).

[33] X. Ouyang, P.L. Varghese, Appl. Opt. 28, 1538 (1989). 
[34] L. Joly, V. Zéninari, B. Parvitte, D. Weidmann, D. Courtois, Y. Bonetti, T. Aellen, M. Beck, J. Faist, D. Hofstetter, Appl. Phys. B 77, 703 (2003).

[35] V. Zéninari, L. Joly, B. Grouiez, B. Parvitte, A. Barbe, JQSRT 105, 312 (2007).

[36] R. A. Toth, L. R. Brown, C. Plymate, JQSRT 59, 529 (1998). 
Table 1. List of the dimensionless parameters of the normalized line shape functions.

\begin{tabular}{|c|c|c|}
\hline \multicolumn{2}{|c|}{ Dimensionless Parameter } & \multirow{2}{*}{ Related Dimensional parameters ${ }^{b}$} \\
\hline Definition $^{a}$ & Description & \\
\hline$x=\frac{v-v_{0}}{\alpha_{D}}$ & $\begin{array}{l}\text { Frequency detuning from } \\
\text { resonance frequency }\end{array}$ & $\begin{array}{l}\text { Wavenumber frequency } v \text { and line } \\
\text { centre } v_{0}\left[\mathrm{~cm}^{-1}\right] \text {. }\end{array}$ \\
\hline$y=\frac{\gamma_{L}}{\alpha_{D}}$ & $\begin{array}{l}\text { Frequency of broadening } \\
\text { collisions }\end{array}$ & $\begin{array}{l}\text { Collisional half width, } \gamma_{L} \text {; Pressure } \\
\text { broadening coefficient, } \gamma_{L}^{0}\left[\mathrm{~cm}^{-1} \mathrm{~atm}^{-1}\right]\end{array}$ \\
\hline$z=\frac{\beta}{\alpha_{D}}$ & $\begin{array}{l}\text { Effective frequency of } \\
\text { velocity changing collisions } \\
\text { (weak collision model) }\end{array}$ & $\begin{array}{l}\text { Collisional narrowing, } \beta ; \text { Collisional } \\
\text { narrowing parameter, } \beta^{0}\left[\mathrm{~cm}^{-1} \mathrm{~atm}^{-1}\right] .\end{array}$ \\
\hline$\zeta=\frac{\Omega}{\alpha_{D}}$ & $\begin{array}{l}\text { Frequency of velocity } \\
\text { changing collisions } \\
\text { (strong collision model) }\end{array}$ & $\begin{array}{l}\text { Collisional narrowing, } \Omega ; \text { Collisional } \\
\text { narrowing parameter, } \Omega^{0}\left[\mathrm{~cm}^{-1} \mathrm{~atm}^{-1}\right] \text {. }\end{array}$ \\
\hline$s=\frac{\delta}{\alpha_{D}}$ & $\begin{array}{l}\text { Effective frequency of } \\
\text { shifting collision }\end{array}$ & $\begin{array}{l}\text { Line shift, } \delta \text {, Line shift coefficient, } \delta^{0} \\
{\left[\mathrm{~cm}^{-1} \mathrm{~atm}^{-1}\right] .}\end{array}$ \\
\hline
\end{tabular}

${ }^{a} \alpha_{D}$ denotes the 1/e Doppler half width.

${ }^{b}$ The upper script 0 indicates quantities normalized to a pressure of $1 \mathrm{~atm}$. 
Table 2. Normalized line shape function implemented in VLSFP.

Profile and Physical features

Equation $^{a}$

\section{Doppler}

Inhomogeneous broadening by random thermal motion.

\section{Lorentz}

Homogeneous broadening by internal state perturbing collisions with negligible thermal motion

$$
f(x-s, y)=\frac{1}{\pi} \frac{y}{y^{2}+(x-s)^{2}}
$$

contribution.

\section{Voigt}

Broadening by thermal motion and internal state perturbing collisions. The two broadening mechanism $f(x-s, y)=\operatorname{Re}[w(x, y)]=\frac{y}{\alpha_{D} \pi^{3 / 2}} \int_{-\infty}^{+\infty} \frac{\mathrm{e}^{-t^{2}}}{[(x-s)-t]^{2}+y^{2}} d t$ are considered to be statistically independent.

\section{Galatry}

Broadening by thermal motion and internal state perturbing collisions; weak collision model for velocity

$$
f(x-s, y, z)=\frac{1}{\pi} \operatorname{Re}\left\{\int_{0}^{+\infty} \mathrm{e}^{-t[y+\mathrm{i}(x-s)]+\left(2 z^{2}\right)^{-1}\left[1-z t-\mathrm{e}^{-z t}\right]} d t\right\}
$$
changing collisions.

\section{Nelkin \& Ghatak / Rautian \&}

\section{Sobel'man}

Broadening by thermal motion and internal state perturbing collisions;

$$
f(x-s, y, \zeta)=\frac{1}{\sqrt{\pi}} \operatorname{Re}\left[\frac{w(x-s, y+\zeta)}{1-\sqrt{\pi \zeta w(x-s, y+\zeta)}}\right]
$$
strong collision model for velocity changing collisions.

\footnotetext{
${ }^{a} w(x, y)$ is the complex probability function.
} 
Table 3. Obtained line parameters of the ro-vibrational transitions of the $v_{1}$ band of $\mathrm{SO}_{2}{ }^{a}$ employed to test VLSFP.

\begin{tabular}{|c|c|c|c|c|c|c|c|c|c|c|}
\hline \multirow{2}{*}{$J^{\prime}$} & \multirow{2}{*}{$K_{a}^{\prime}$} & \multirow{2}{*}{$K_{c}^{\prime}$} & \multirow{2}{*}{$J^{\prime \prime}$} & \multirow{2}{*}{$K_{a} "$} & \multirow{2}{*}{$K_{c}^{\prime \prime}$} & \multirow{2}{*}{$\tilde{\mathbf{v}}_{0}\left[\mathrm{~cm}^{-1}\right]$} & \multicolumn{2}{|c|}{$\gamma_{\text {self }}^{0}\left[\mathbf{c m}^{-1} \mathbf{a t m}^{-1}\right]$} & \multicolumn{2}{|c|}{$S^{0}\left[10^{-22} \mathrm{~cm} \cdot\right.$ molecule $\left.^{-1}\right]$} \\
\hline & & & & & & & This work & Literature & This work & Literature \\
\hline 20 & 14 & 6 & 21 & 15 & 7 & $1089.52139(7)$ & $0.266(5)$ & $0.26_{4}\left(1_{4}\right)^{b}$ & $5.51(3)$ & $5.67(21)^{b}$ \\
\hline 33 & 11 & 23 & 34 & 12 & 22 & $1089.72440(9)$ & $0.340(10)$ & $0.29_{9}\left(1_{7}\right)^{b}$ & $3.55(6)$ & $2.99(18)^{b}$ \\
\hline 29 & 18 & 12 & 29 & 19 & 11 & $1089.79423(13)$ & $0.20(2)$ & $0.21_{7}\left(1_{1}\right)^{c}$ & $0.65(2)$ & $0.746(101)^{d}$ \\
\hline 51 & 7 & 45 & 52 & 8 & 44 & $1089.8234(2)$ & $0.40(6)$ & - & $0.51(5)$ & - \\
\hline 24 & 13 & 11 & 25 & 14 & 12 & $1089.84713(3)$ & $0.268(7)$ & $0.28_{3}\left(1_{3}\right)^{c}$ & $5.70(5)$ & $5.66(11)^{d}$ \\
\hline 15 & 15 & 1 & 16 & 16 & 0 & $1089.87257(4)$ & $0.161(7)$ & $0.19_{2}\left(1_{0}\right)^{c}$ & $6.78(8)$ & $6.91(12)^{d}$ \\
\hline 32 & 10 & 22 & 33 & 11 & 23 & $1089.7409(2)^{e}$ & $0.27(4)$ & $0.25_{0}\left(2_{1}\right)^{c}$ & $0.71(4)$ & $0.563(12)^{d}$ \\
\hline
\end{tabular}

${ }^{a}$ Figures in parentheses are one standard deviations.

${ }^{b}$ From Ref. [34].

${ }^{c}$ From Ref. [35]; the quoted uncertainties have been derived from the error bars of Figures 4, 6 and 8 .

${ }^{d}$ From Ref. [35].

${ }^{e}$ Belonging to $v_{1}+v_{2}-v_{2}$ of ${ }^{32} \mathrm{SO}_{2}$; an overlapping with $16_{13,3} \leftarrow 17_{14,4}$ of ${ }^{34} \mathrm{SO}_{2} v_{1}$ band also occurs. 
Table 4. Obtained line parameters of the ro-vibrational transitions of the $v_{7}$ band of $\mathrm{CH}_{2} \mathrm{CHF}^{a}$.

\begin{tabular}{|c|c|c|c|c|c|c|c|c|c|}
\hline \multirow{2}{*}{$J^{\prime}$} & \multirow{2}{*}{$K_{a}^{\prime}$} & \multirow{2}{*}{$K_{c}^{\prime}$} & \multirow{2}{*}{$J^{\prime \prime}$} & \multirow{2}{*}{$K_{a}^{\prime \prime}$} & \multirow{2}{*}{$K_{c}^{\prime \prime}$} & \multirow{2}{*}{$\tilde{\mathbf{v}}_{0}\left[\mathbf{c m}^{-1}\right]$} & \multicolumn{2}{|c|}{$\gamma_{\text {self }}^{0}\left[\mathrm{~cm}^{-1} \mathbf{a t m}^{-1}\right]$} & \multirow{2}{*}{$S^{0}\left[10^{-21} \mathrm{~cm} \cdot \mathrm{molecule}{ }^{-1}\right]$} \\
\hline & & & & & & & Voigt & Nelkin - Ghatak & \\
\hline 10 & 1 & 10 & 11 & 1 & 11 & $1148.1499(8)$ & $0.80(10)$ & $0.78(2)$ & $2.8(2)$ \\
\hline 10 & 7 & 3 & 11 & 7 & 4 & $1148.1963(4)$ & - & $0.69(6)$ & $3.05(4)$ \\
\hline 10 & 8 & 2 & 11 & 8 & 3 & $1148.30268(13)$ & $0.48(4)$ & $0.88(4)$ & $2.88(2)$ \\
\hline 9 & 1 & 8 & 10 & 1 & 9 & $1148.3454(2)$ & $0.74(2)$ & $0.80(2)$ & $4.253(13)$ \\
\hline 8 & 0 & 8 & 9 & 1 & 9 & $1148.3689(2)$ & - & - & $0.72(2)$ \\
\hline 10 & 9 & 1 & 11 & 9 & 2 & $1148.4210(2)$ & $0.31(2)$ & $0.54(6)$ & $2.59(2)$ \\
\hline 9 & 2 & 7 & 10 & 2 & 8 & $1148.4327(2)$ & $0.75(2)$ & $0.39(3)$ & $4.29(2)$ \\
\hline 9 & 1 & 8 & 9 & 2 & 7 & $1148.4574(4)$ & $0.51(5)$ & $0.87(10)$ & $0.63(3)$ \\
\hline 20 & 1 & 19 & 20 & 2 & 18 & $1148.5396(3)$ & - & - & $0.70(4)$ \\
\hline 10 & 10 & 0 & 11 & 10 & 1 & $1148.5513(3)$ & - & - & $0.85(2)$ \\
\hline 9 & 3 & 6 & 10 & 3 & 7 & $1148.5644(3)$ & $0.47(6)$ & $0.45(5)$ & $3.30(5)$ \\
\hline 9 & 3 & 7 & 10 & 3 & 8 & $1148.5742(3)$ & - & $0.65(10)$ & $4.6(2)$ \\
\hline 9 & 2 & 8 & 10 & 2 & 9 & $1148.5792(3)$ & - & $0.84(6)$ & $5.3(3)$ \\
\hline
\end{tabular}

${ }^{a}$ Figures in parentheses are one standard deviations. 


\section{Figure Captions}

Figure 1. Block diagram representing the implementation of VLSFP. Each box represents a class referred to as Object (C. M. stands for collision model). The objects interact with each other to make the application: the arrow means "derives from", the diamond means "makes use of" (see text for discussion).

Figure 2. A single line fit obtained employing VLSFP. Row (a) shows the experimental (O) and computed spectrum (-). Experimental details: $\mathrm{SO}_{2}$ total pressure $=107.0 \mathrm{~Pa}$, path length $=92.3 \mathrm{~cm}$, temperature $=297$ K. The remaining rows show the residuals obtained from different models: (b) Voigt; (c) weak collision model (wcm); (d) strong collision model $(\mathrm{scm})$. The standard deviations of the fits, $\sigma\left(\mathrm{cm}^{-1}\right)$, are: $\sigma_{\mathrm{Voigt}}=$ $1.6 \times 10^{-3} ; \sigma_{\mathrm{wcm}}=0.88 \times 10^{-3} ; \sigma_{\mathrm{scm}}=0.90 \times 10^{-3}$.

Figure 3. Multi - line fit of the $\mathrm{SO}_{2}$ spectrum between 1089.69 and $1089.87 \mathrm{~cm}^{-1}$ : row (a) shows the experimental $(\bigcirc)$ and computed spectrum (-). Experimental details: $\mathrm{SO}_{2}$ total pressure $=511.4 \mathrm{~Pa}$, path length $=92.3$ $\mathrm{cm}$, temperature $=297 \mathrm{~K}$. The remaining rows show the residuals obtained from different models: (b) Voigt; (c) weak collision model (wcm); (d) strong collision model ( $\mathrm{scm})$. The standard deviations of the fits, $\sigma\left(\mathrm{cm}^{-1}\right)$, are: $\sigma_{\mathrm{Voigt}}=4.0 \times 10^{-3} ; \sigma_{\mathrm{wcm}}=3.1 \times 10^{-3} ; \sigma_{\mathrm{scm}}=3.2 \times 10^{-3}$. 
Figure 4. Comparison between the determined line parameters $(\nabla)$ and the values of Refs. [34] ( $\diamond)$ and [35] ( $\bigcirc)$. Upper trace: self broadening coefficients; lower trace: line strengths.

Figure 5. Vinyl fluoride spectrum between 1148.14 and $1148.6 \mathrm{~cm}^{-1}$ : (a) experimental $(\bigcirc)$ and computed spectrum (-). Experimental details: $\mathrm{CH}_{2} \mathrm{CHF}$ total pressure $=30.6 \mathrm{~Pa}$, path length $=92.3 \mathrm{~cm}$, temperature $=$ 299 K. The remaining rows show the residuals obtained from different models: (b) Voigt; (c) strong collision model (scm); The standard deviations of the fits, $\sigma\left(\mathrm{cm}^{-1}\right)$, are: $\sigma_{\text {Voigt }}=4.5 \times 10^{-3} ; \sigma_{\mathrm{scm}}=2.6 \times 10^{-3}$. 